\title{
Synchronous Detection for Robust 3-D Shape Measurement against Interreflection and Subsurface Scattering
}

\author{
Tatsuhiko Furuse, Shinsaku Hiura ${ }^{\star}$, and Kosuke Sato \\ Graduate School of Engineering Science, Osaka University \\ 1-3 Machikaneyama, Toyonaka, Osaka 560-8531 Japan
}

\begin{abstract}
Indirect reflection component degrades the preciseness of 3-D measurement with structured light projection. In this paper, we propose a method to suppress the indirect reflection components by spatial synchronous detection of structured light modulated with MLS (Maximum Length Sequence, M-sequence). Our method exploits two properties of indirect components; one is the high spatial frequency component which is attenuated through the scattering of projected light, and the other is the geometric constraint between projected light and its corresponding pixel of camera.

Several experimental results of measuring translucent or concave objects show the advantage of our method.
\end{abstract}

Keywords: Subsurface Scattering, Interreflection, Shape Measurement.

\section{Introduction}

In this paper, we present a novel method to precisely measure the 3-D shape of object by suppressing subsurface scattering and interreflection which disturb the correct detection of structured light projected on the object.

In principle, triangulation method using structured light projection detects the point of reflection where the incoming light firstly reaches to the surface of the object. In other words, the method assumes that the directly illuminated part only is brightly shining, or otherwise dark. However, in general, the luminance of the scene consists of not only direct but also indirect reflection component (Figure1). Direct reflection component is the ideal light for 3-D measurement because the line of sight intersects with the incoming light beam exactly on the object surface. On the other hand, indirect component does not directly correspond to the shape of the object and may cause errors.

Indirect component can be classified to subsurface scattering and interreflection. Subsurface scattering is the reflection observed as blurring of light on the surface. Incoming light penetrates into a translucent object, and scattered by particles such as pigments. Therefore, the light exits the surface at a point different from the entrance, and introduces error to measured shape as shown in Figure 1 Interreflection is a phenomenon that the light reflected on the object act as a second light source illuminating the other surface, and produces spurious shape apart from the true surface.

\footnotetext{
${ }^{\star}$ Now at Graduate School of Information Sciences, Hiroshima City University.
} 


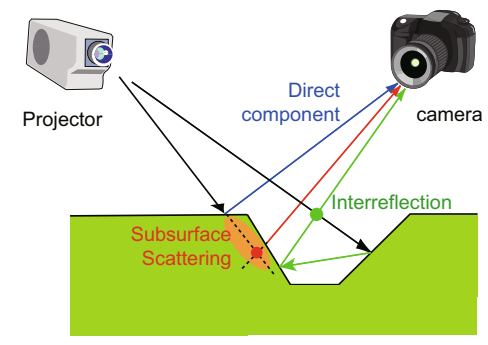

Fig. 1. Error caused by subsurface scattering and interreflection. Subsurface scattering introduces a displacement of measured shape (red dot). Interreflection also generates a spurious shape (green dot) apart from the true surface.

In general, more precisely we want to measure the detail of object, the more strong influence we have via subsurface scattering and interreflecion, even if the object seems opaque in macroscopic scale. For example, a material which attenuates the light by half in $1 \mathrm{~mm}$ on its pathway does not affect to the measurement whose resolution is $10 \mathrm{~mm}$, but may have an influence for the case of requirement of $0.1 \mathrm{~mm}$ resolution. Most nonmetallic objects such as plastic, cloth, paper and wood have more or less subsurface scattering. Similarly, the intensity of interreflection gets higher if the distance between two interacting surfaces is small. Therefore, suppression of such indirect components is important for precise shape measurement in industry and digital archives.

\subsection{Related Works}

Subsurface scattering have been well addressed in computer graphics area. Jensen et al. [6] proposed a mathematical model of subsurface scattering to represent BSSRDF (Bidirectional Surface Scattering Reflectance Distribution Function) with parametric function, and many techniques to render photorealistic images of such objects have been proposed.

In recent years, 3-D measurement of non-lambertian object such as transparent, translucent or specular object have extensively attacked [4]. As described before, subsurface scattering is the main issue to measure the translucent object using structured light. Godin et al. [2] analyzed the defocus and noise of penetrating light by projecting spot light onto a marble stone, because statues made of marble stones are need to be measured in digital archiving area [7] . Goesele et al. [3] measured the shape of translucent object by using laser light and HDR (High Dynamic Range) camera. However, it takes very long time to measure the whole object because it is based on spot light projection method.

Methods for suppressing the influence of indirect components have been proposed. T.Chen et al. [1] improved the phase shifting method by using polarization filter to suppress indirect components. Contrary, Nayar et al. [10] proposed a method to separate direct and indirect reflection component by projecting high spatial frequency pattern onto the object. Since intensity via indirect component receives contributions from various part of projected light, high spatial frequency components tend to be attenuated 
through the light transport of indirect reflection. Using this principle, Mukaigawa et al. [9] analyzed the light transport in homogeneous scattering media using similar high spatial frequency stripe pattern.

Our method is basically based on Nayar's method, but their method is not effective for specular interreflection caused by smooth surface. Therefore we extend the method with MLS (Maximum Length Sequence, M-sequence) which is used for checking the geometric constraint (epipolar constraint) between projector and camera. Generally, observed light via specular interreflection does not satisfy the geometric constraint, and synchronous detection effectively suppress the component.

Synchronous detection techniques are widely used in control engineering, communication technologies and weak signal detection. For example, CDMA (Code Division Multiple Access) uses pseudorandom sequence such as MLS to separate multiplexed signals or suppressing multi-path fading effect caused by reflected signals in temporal domain. The principle of proposed method is similar from the mathematical point of view, but ours is not temporal but spatial (or geometric).

\section{Surpression of Indirect Component Using Spatially Modulated Light}

In this section, we will describe the method to suppress the indirect component of projected light using spatially modulated light. Firstly we will start from an extension of slit light projection method, then apply the principle to the Gray-code projection method.

\subsection{Spatially Modulated Slit Light}

MLS (Maximum Length Sequence, M-sequence) is a pseudorandom binary sequence which has three advantages for our purpose. Firstly, MLS contains high frequency component which is more likely to be attenuated through indirect light transport. The second advantage is that MLS has high autocorrelation value only if the phase difference is zero. MLS is also easy to implement because it has only two binary values, therefore no photometric calibration is necessary for projector. To suppress the indirect component of projected light, we spatially modulate the light along the slit direction using MLS as shown in Figure 2, The intensity distribution of projected pattern $L\left(x_{p}, y_{p}, t\right)$ is denoted as

$$
L\left(x_{p}, y_{p}, t\right)=\left\{\begin{array}{rr}
M\left(\left(t-x_{p}\right)\right. & \bmod T) y_{p}=\bar{y}_{p} \\
0 & y_{p} \neq \bar{y}_{p}
\end{array}\right.
$$

where $T$ is the cycle of MLS function $M(t)$, and $\left(x_{p}, y_{p}\right)$ is the coordinate on the projector image. The slit light is parallel to the axis $x_{p}$, and the position of the slit is determined by $\bar{y}_{p}$. The MLS pattern is shifted along the slit light for one cycle, therefore $T$ different pattern is projected for each slit position.

The image of the scene is captured by the camera as shown in Figure 2. In prior to the measurement, the geometric relationships between projector and camera are calibrated using a reference object. This process is common to the calibration of quantitative 3$\mathrm{D}$ measurement, therefore this process does not lose the feasibility at all. From the 


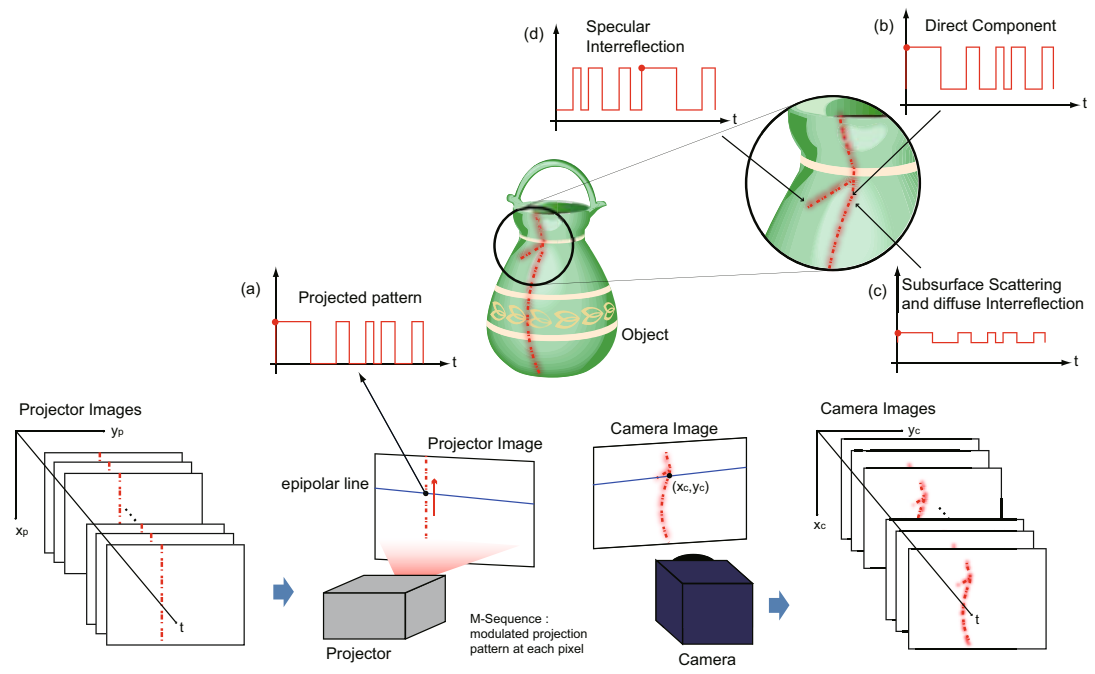

Fig. 2. Schematic illustration of proposed method. Slit light is modulated with MLS code, and captured by a camera. The amplitude of intensity by subsurface scattering is very low, and the phase of the intensity sequence by interreflection is shifted.

calibration parameters of camera and projector, we can derive the following epipolar constraint equation,

$$
\left(\begin{array}{lll}
x_{p} & y_{p} & 1
\end{array}\right) F\left(\begin{array}{c}
x_{c} \\
y_{c} \\
1
\end{array}\right)=0
$$

where $F$ is called fundamental matrix [8]. Using this equation, we can calculate the epipolar line on the projector image for each pixel $\left(x_{c}, y_{c}\right)$ on camera image. The epipolar line has a intersection with the slit light, and we can decide the corresponding phase of MLS of projected light for each point on the image. More specifically, we can determine the corresponding coordinate $\left(x_{p}, \bar{y}_{p}\right)$ on the projector image by

$x_{p}=-\frac{e_{2} \bar{y}_{p}+e_{3}}{e_{1}}$ where

$$
\left(\begin{array}{l}
e_{1} \\
e_{2} \\
e_{3}
\end{array}\right)=F\left(\begin{array}{l}
x_{c} \\
y_{c} \\
1
\end{array}\right) .
$$

The process of synchronous detection of modulated slit light is illustrated in Figure 3 We simply calculate the correlation between binary value of projected MLS pattern and intensity sequence from the camera image, then use the correlation value for slit detection instead of the raw intensity on the image.

As described in the paper [10], high spatial component in projected light is more likely to be attenuated by the propagation of subsurface scattering or diffuse interreflection. However, specular interreflection preserves the structure of projected light, therefore, frequency analysis is not sufficient to suppress the effect. Fortunately, specular interreflection can be assumed as a light projected from the other point, therefore, the 


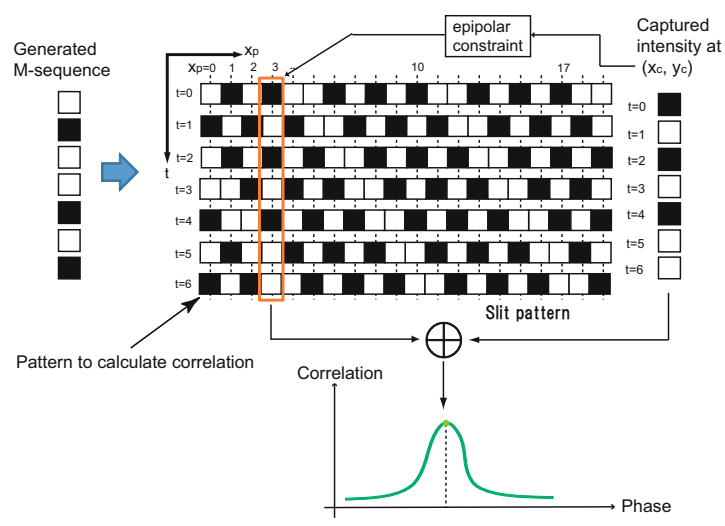

Fig. 3. Synchronous detection of captured intensity using geometric constraint

epipolar constraint is no longer satisfied. In the next section, we will show the faster method based on Gray code projection.

\subsection{Modulation of Gray Code Pattern}

As shown in the experimental results below, proposed method with synchronous detection using modulated slit light is effective for suppressing indirect component. However, it takes much time to measure the whole shape of the object. The number of projection pattern and corresponding image capture is $T \cdot N$ where $\mathrm{T}$ is the cycle of MLS and $N$ is the number of slit planes which corresponds to the depth resolution. Actually in our experiment, we used parameters of $T=31$ and $N=1024$ which take around 18 minutes if the frame rate of projection and acquisition is 30 frames/sec.

Fortunately, several methods to accelerate the range measurement using structured light have been proposed, and the temporal space coding [5]11] is one of the most successful method using Gray-code. If we use complementary pattern projection 1 , it only needs $2 \log _{2} N$ patterns for measuring the whole object. Therefore we combine our synchronous detection framework with temporal space coding method.

The projection patterns which consist of Gray-code and MLS are illustrated in Figure 4 The geometric synchronous detection requires that the phase of MLS should be uniquely determined by the coordinate on the image plane $\left(x_{c}, y_{c}\right)$. In the case of slit light projection method, the phase of MLS can be uniquely determined because the cross section of slit light and epipolar line is a single point. Contrary to the case of slit-code projection, the bright part in projection pattern has certain width, therefore we should align the lines of MLS code parallel to epipolar lines as shown in Figure 5 This arrangement make the phase corresponds to a epipolar line unique, therefore we can use the same synchronous detection method again.

\footnotetext{
${ }^{1}$ For each bit plane, positive and its inverted pattern are projected, and sign of subtraction of two images is used for binarized image.
} 


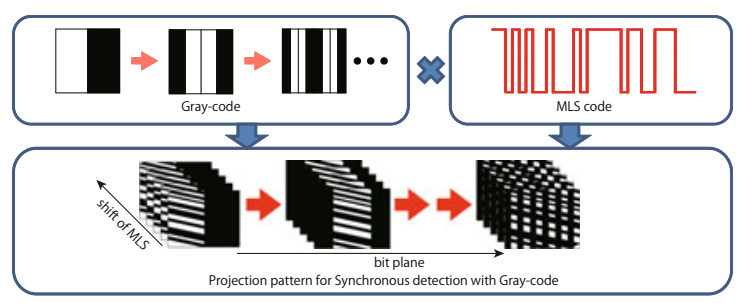

Fig. 4. Modulated Gray-code pattern for temporal space coding method with synchronous detection
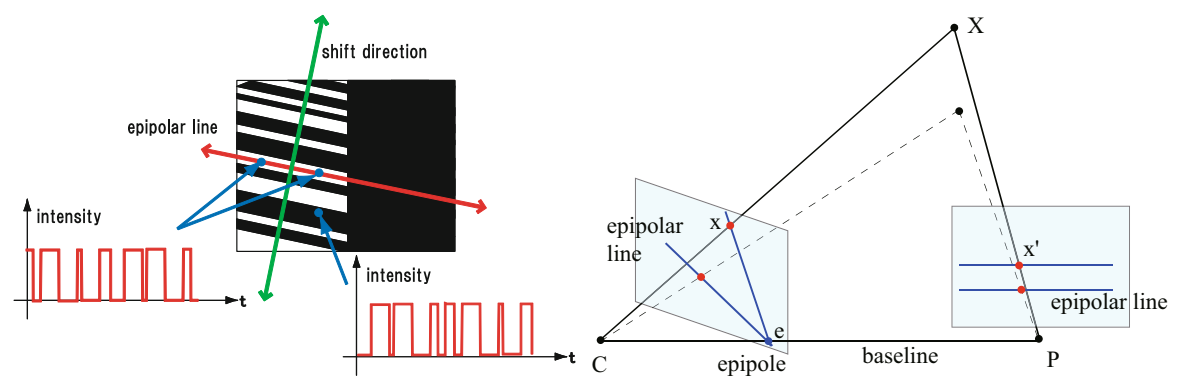

Fig. 5. Arrangement of MLS code in the projection pattern. Arrangement of camera and projector. If the image plane of the projector is parallel to the baseline, all epipolar line on the projector image is parallel.

IF the epipolar lines are not parallel each other, the density of the code is not homogeneous. However, the density of the pattern decides the performance of eliminating indirect component. Therefore, we arrange the image plane of the projector parallel to the baseline as shown in Figure 5, In this case, the all epipolar lines on the projector image are parallel. Contrary, the epipolar lines on the camera image are not necessary to be aligned parallel.

By using the temporal space coding method with synchronous detection, the total number of projected pattern is $2 T \log _{2} N$. The condition of $T=31$ and $N=1024$ takes only 21 seconds with frame rate of 30 frames/sec. This is not only 50 times faster than the case of slit light projection method with synchronous detection, but also even faster than simple slit light projection.

\section{Experiments}

The appearance of experimental setup is shown in Figure 7 We used a liquid crystal projector (EPSON EMP-1710, resolution : $1024 \times 768$ pixel) with a convex lens which focal length is $330 \mathrm{~mm}$. The convex lens is used to adjust the focus on the object at short distance. The cycle of used MLS code is $T=31$, and the calibration of the system is done in prior to the measurement using a reference object. 


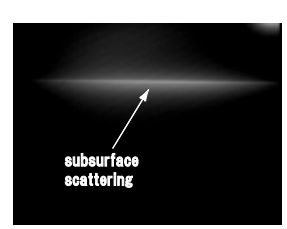

(a)Intensity

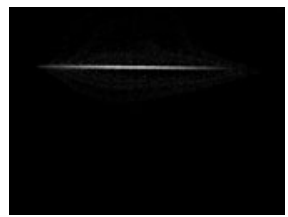

(b)Correlation value

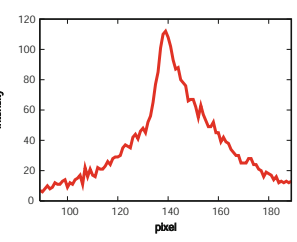

(c)Intensity

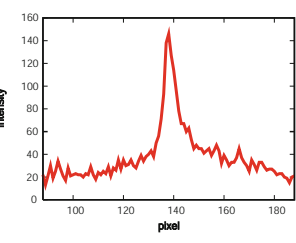

(d)Correlation value

Fig. 6. Observed slit light under subsurface scattering
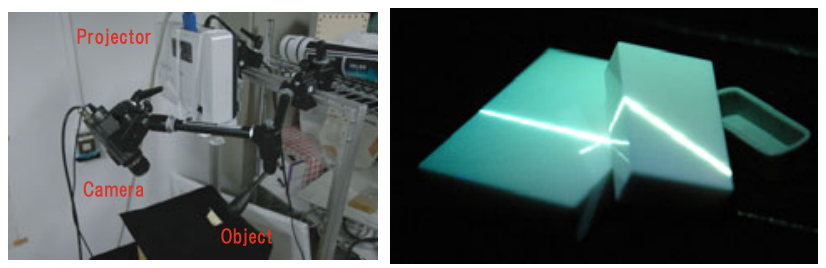

(a)Experimental setup and arrangement of objects for evaluation

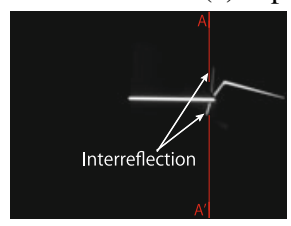

(b)Intensity

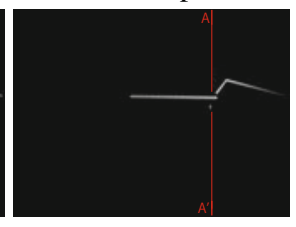

(c)Correlation value

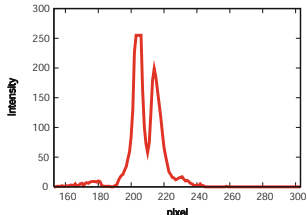

(d)Intensity

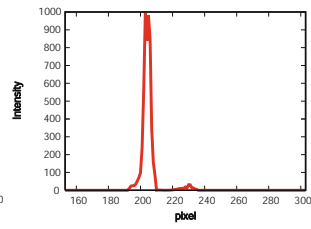

(e)Correlation value

Fig. 7. Observed slit light under interreflection

\subsection{Suppression of Indirect Component}

In prior to showing the performance of shape measurement, we will show the result of suppressing indirect component in this section. Figure 6 is a result of suppressing subsurface scattering using an opalescent acrylic plate. Figure 6a) and (b) show the intensity distribution of normal and synchronous detection respectively. The intensity on a vertical line at the center of Figure (a) and (b) are shown in Figure 6(c) and (d) respectively. The half-value widths of slit light, 21.2 and 6.7 pixels respectively, show that the subsurface scattering is effectively suppressed by our method.

Figure 7 shows the result of suppressing specular interreflection. The object is a stack of zirconia ceramic blocks shown in Figure 7(a). Figure 7(d) and (e) are the intensity distribution on the line A-A' in Figure 7 b) and (c) respectively. False slit is well eliminated by synchronous detection though it is clearly visible in raw intensity image.

\subsection{Shape Measurement with Slit Light Projection}

The results of shape measurements are shown in the last 2 pages. Figure 8 is the result with suppressed interreflection. The object (small figure of a house, width is around $40 \mathrm{~mm}$ ) is measured from the front side of the house. Interreflection causes a false shape 


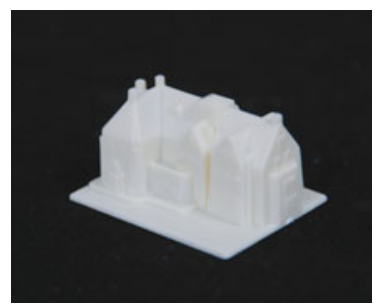

(a)Measured object

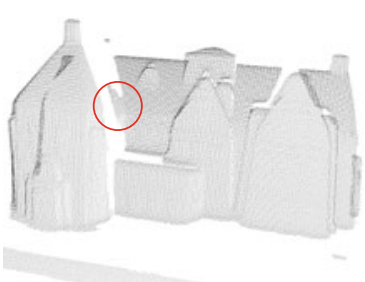

(b)Conventional method

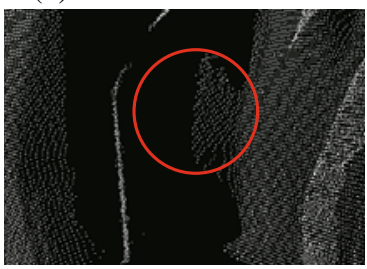

(d)Magnified view of (b)

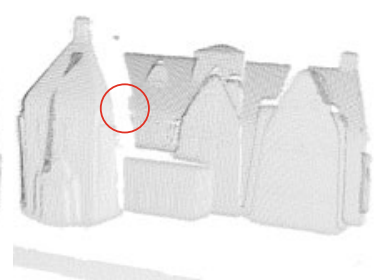

(c)Proposed method

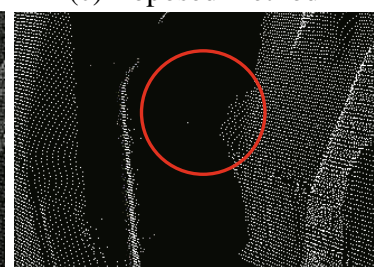

(e)Magnified view of (c)

Fig. 8. Experimental result 1 (slit light projection)

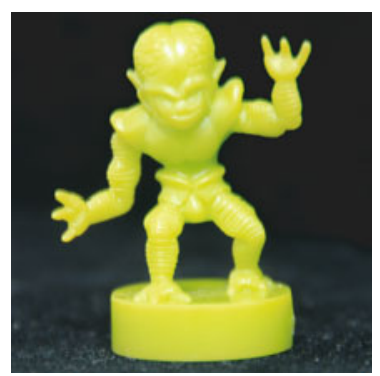

(a)Measured object

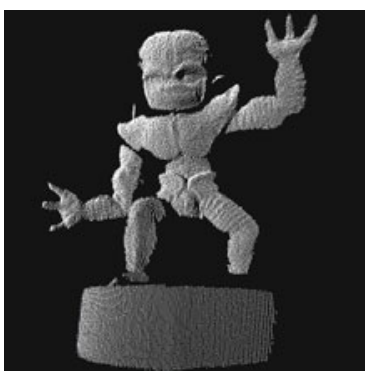

(b)Conventional method

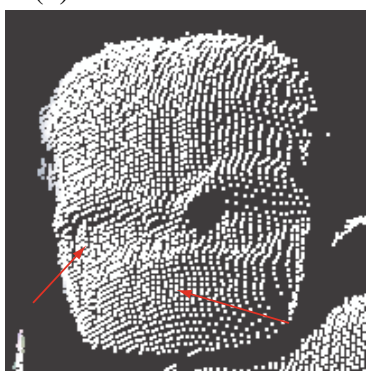

(d)Magnified view of (b)

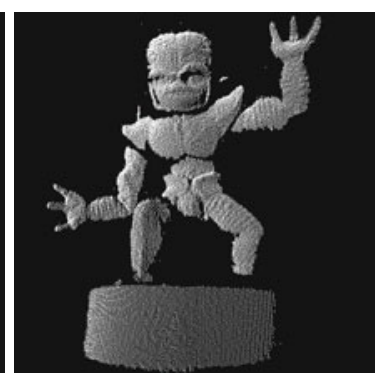

(c)Proposed method

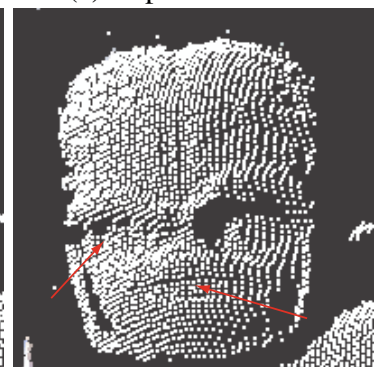

(e)Magnified view of (c)

Fig. 9. Experimental result 2 (slit light projection)

(indicated by a red circle) with conventional method, and it is well suppressed by the proposed method.

The result with translucent object with subsurface scattering is shown in Figure 9 Since the figure is so small (height is around $38 \mathrm{~mm}$ ), some details of the figure are lost 


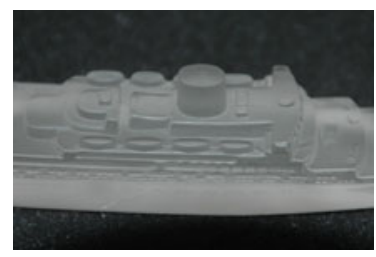

(a)Measured object

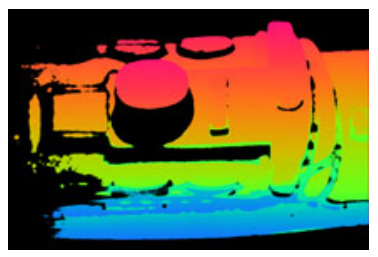

(b)Conventional method

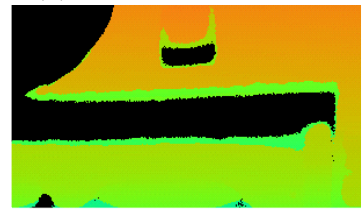

(d)Magnified view of (b)

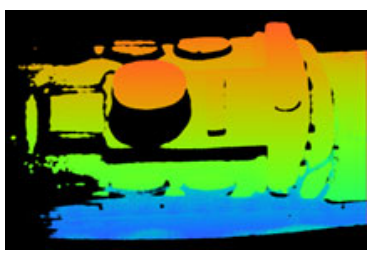

(c)Proposed method

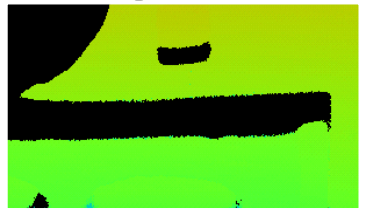

(e)Magnified view of (c)

Fig. 10. Experimental result 1 (temporal space coding)

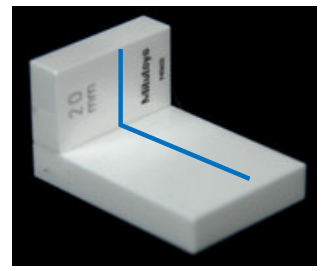

(a)Measured object

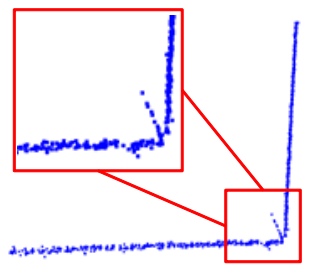

(b)Conventional method

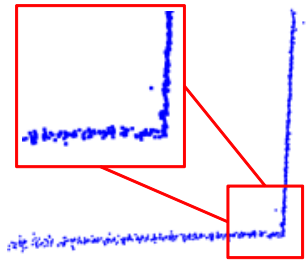

(c)Proposed method

Fig. 11. Experimental result 2 (temporal space coding)

with conventional method. As shown in Figure 9(d), the edges around the mouse and eyes are almost lost, but the proposed method well preserves the edge.

\subsection{Shape Measurement with Gray-Code Projection}

Indirect component of structured light causes not only displacement of measured shape but also spurious shape by decoding error for temporal space coding method. Figure 10 shows the result of measuring a figure of a ship made of flosted glass. Figure 10 b) - (e) shows the color map of measured height of obliquely placed object. At the edges of flat part, false step shape is observed with conventional method shown in Figure 10 b) and (d). Contrary, the shape measured with proposed method has no false step shape around the edge as shown in Figure 10 (c) and (e).

To show the result of suppressing specular interreflection, we used a stack of ceramic blocks again as shown in Figure 11. Figure 11 (b) shows that conventional method produces a spurious shape around the concave edge of the object, and the shape of the edge is not acute but rounded. Contrary, with our proposed method, the shape of the object is properly measured as shown in Figure 11(c).

The main contribution of our method is to suppress the erroneous result caused by indirect components. The amount of error much depends on the shape and material of 
the object, and the other conditions such as the angle of incident light also heavily affect to the result. Therefore, it is not adequate to show a quantitive comparison between conventional and proposed method with measured shape. Instead, we showed the effect of our method with the difference of intensity distribution in section 3.1

\section{Conclusion}

We proposed the 3-D shape measurement method robust against both interreflection and subsurface scattering. MLS pattern is very useful to combine two suppression principles based on geometric constraint and transfer characteristics in spatial frequency. Experimental results showed the practicality of our method for translucent or shiny object with fine details.

Since the false image caused by the interreflection sometimes has exactly same phase as the true one, multiple cameras or projectors should be effective to verify the true image using multiple epipolar lines. Synchronous detection technique will be also useful for disambiguating simultaneous projections from multiple projectors.

\section{References}

1. Chen, T., Lensch, H.P.A., Fuchs, C., Seidel, H.P.: Polarization and phase-shifting for $3 \mathrm{~d}$ scanning of translucent objects. In: Proc. CVPR, pp. 1-8 (2007)

2. Godin, G., Rioux, M., Beraldin, J.A.: An assessment of laser range measurement on marble surfaces. In: Conference on Optical 3D Measurement Techniques, pp. 49-56 (2001)

3. Goesele, M., Lensch, H.P.A., Lang, J., Fuchs, C., Seidel, H.P.: Disco - acquisition of translucent object. In: Proc. SIGGRAPH, pp. 835-844 (2004)

4. Ihrke, I., Kutulakos, K.N., Lensch, H.P.A., Magnor, M., Heidrich, W.: State of the art in transparent and specular object reconstruction. In: Proc. Eurographics, pp. 1-22 (2008)

5. Inokuchi, S., Sato, K., Matsuda, F.: Range-imaging system for $3 \mathrm{~d}$ object recognition. In: Proc. of 7th International Conference on Pattern Recognition, pp. 806-808 (1984)

6. Jensen, H.W., Marschner, S.R., Levoy, M., Hanrahan, P.: A practical model for subsurface light transport. In: Proc. SIGGRAPH, pp. 511-518 (2001)

7. Levoy, M., Pulli, K., Curless, B.: Digital michelangelo project: $3 \mathrm{~d}$ scanning of large statues. In: Proc. SIGGRAPH, pp. 131-144 (2000)

8. Luong, Q.T., Faugeras, O.D.: The fundamental matrix: Theory, algorithms, and stability analysis. International Journal of Computer Vision 17(1), 43-75 (1996)

9. Mukaigawa, Y., Yagi, Y., Raskar, R.: Analysis of light transport in scattering media. In: Proc. CVPR, pp. 153-160 (2010)

10. Nayar, S.K., Krishnan, G., Grossberg, M., Raskar, R.: Fast separation of direct and global components of a scene using high frequency illumination. In: Proc. SIGGRAPH, pp. 93509943 (2006)

11. Sato, K., Inokuchi, S.: Range-imaging system utilizing nematic liquid crystal mask. In: Proc. of 1st International Conference on Computer Vision, pp. 657-661 (1987) 